\title{
Corrección de mordida cruzada posterior vestibular en dentición primaria. Reporte de dos casos clínico
}

\author{
Jerusalén E. Mata T. ${ }^{1}$ \\ A. Carolina Medina ${ }^{2}$, \\ María del Carmen Prieto ${ }^{3}$.
}

\section{Resumen}

La mordida cruzada posterior vestibular cruzada bucal o mordida en tijera ocurre cuando las cúspides linguales de los molares superiores ocluyen vestibularmente a las fosas centrales de los molares inferiores o cuando las caras palatinas de los dientes superiores están en contacto con las caras vestibulares de los dientes inferiores. Pueden ser funcionales, causadas por inclinación de los molares inferiores hacia lingual o por asimetrías mandibulares. El tratamiento temprano es recomendado para normalizar las relaciones transversales y promover un correcto desarrollo de la oclusión. Se presentan dos casos de mordida cruzada posterior vestibular unilateral en dentición primaria y mixta temprana. Caso 1: paciente masculino de 5 años de edad, presentó lesiones extensas de caries, normoclusión, mordida cruzada posterior vestibular unilateral derecha y fue tratado me- diante aparato fijo tipo Bi-Helix con bandas cementadas a los segundos molares primarios. Caso 2: paciente masculino de 5 años de edad, presentó lesiones extensas de caries, normoclusión y mordida cruzada posterior vestibular unilateral Izquierda. Fueron colocadas pistas directas de resina. En ambos casos se logró una relación trasversal satisfactoria, corrigiendo la mordida cruzada vestibular. Conclusión: La mordida cruzada posterior vestibular puede presentarse debido a desviación mandibular por interferencias oclusales y a inclinaciones inapropiadas de los molares primarios. Cuando esta es la etiología, el tratamiento es exitoso al utilizar pistas directas de resina, eliminación de contactos prematuros y expansor inferior tipo Bi-Helix.

Palabras claves: Mordida cruzada, dentición primaria, dentición mixta, maloclusiones, Odontopediatría.

Relato de caso

\section{Correcção de mordida cruzada vestibular em dentição decídua. Relato de dois casos.}

\section{Resumo}

A mordida cruzada posterior vestibular ou mordedura em tesoura acontece quando as cúspides linguais dos molares superiores ocluem para vestibular das fossas centrais dos molares inferiores, ou quando as faces palatinas dos dentes superiores ficam em contacto com as faces vestibulares dos dentes inferiores. Podem ser funcionais ou ser causadas por inclinações lingual dos molares inferiores ou por assimetrias mandibulares. O tratamento precoce é recomen-

1. Especialista en Odontología Infantil UCV. El Encantado Humboltd Caracas Venezuela.

2. Especialista en Odontología Infantil, MSc en Odontología, Profesor Titular, Departamento de Odontopediatría y Ortodoncia, Facultad de Odontología, UCV

3. Especialista en Odontología Infantil, Profesor Asistente adscrito a la Cátedra de Odontología Pediátrica, Miembro del Comité Académico del Postgrado de Odontología Pediátrica, UCV. 
dado para normalizar as relações transversais e promover o correto desenvolvimento da oclusão. São apresentados dois casos de mordida cruzada posterior vestibular unilateral em dentição decídua e mista. Caso 1: paciente do sexo masculino, 5 anos de idade, apresentou lesões extensas de cárie, normoclusão, mordida cruzada posterior vestibular unilateral direita e foi tratado com aparelhos fixo tipo Bi-Helix com bandas cimentadas nos segundos molares decíduos. Caso 2: paciente do sexo masculino, 5 anos de idade, apresentou lesões extensas de cárie, normoclusão, mordida cruzada posterior vestibular unilateral esquerda e foram colocadas pistas diretas em resina. Nos dois casos, foi alcançado uma relação transversal satisfatória, com a correção da mordida cruzada vestibular. Conclusão: a mordida cruzada posterior vestibular pode acontecer devido ao desvio mandibular por interferências oclusais e a inclinações inadequadas aos molares decíduos. Quando esta é a etiologia, o tratamento é bem sucedido com a utilização de pistas directas de resina, eliminação de contatos prematuros e aparelho expansor inferior tipo Bi-Helix.

Plavras chiaves: Mordida cruzada, Dentição decídua, Dentição Mista, Maloclusão, Crianças, Odontopediatria

\section{Review Article}

\section{Posterior buccal crossbite correction in the primary dentition. Report of two cases.}

\begin{abstract}
Posterior vestibular cross bite buccal cross or scissors bite occurs when the lingual cusps of the upper molars occlude buccally to the central pits of the lower molars or when the palatal surfaces of the maxillary teeth are in contact with the buccal surfaces of the lower teeth. They may be functional, caused by a lingual inclination of the lower molars or asymmetries in the mandible. The early treatment is recommended for normalize transversal relations and promote correct development of occlusion. Two cases of vestibular unilateral posterior cross bite in primary and early mixed dentition are presented. Case 1: male patient age 5, presented extensive carious lesions, normocclusion, vestibular unilateral posterior cross bite right side was treated with Bi Helix appliance,
\end{abstract}

bands cemented to the second primary molars. Case 2: male patient age 5, presented extensive carious lesions, normocclusion and vestibular unilateral posterior cross bite left side was treated with direct composite tracks. In both cases was achieved satisfactory transverse relationship by correcting the vestibular cross bite. Conclusion: The posterior vestibular cross bite can occur due to occlusal interferences that produce mandibular deviation and inappropriate inclinations of primary molars. When this is the etiology, using direct composite tracks, elimination of premature contacts and lower expander type bi Helix is a successful treatment.

Keywords: Cross bite, primary dentition, mixed dentition, Malocclusion, children, Pediatric Dentistry. 


\section{Introducción:}

La mordida cruzada posterior, o mordida en tijera ${ }^{1}$

${ }^{2}$ es la relación transversal anormal en la que las cúspides palatinas de los molares superiores ocluyen vestibularmente a las fosas de los molares mandibulares, llegando a contactar con las cúspides vestibulares o las caras vestibulares de los moalres inferiores. Puede involucrar uno o varios dientes del maxilar, la mandíbula o ambos ${ }^{1}$, Pueden ser unilaterales o bilaterales (Síndrome de Brodie). Siendo que en dentición primaria la mayoría de las mordidas cruzadas se observan unilaterales. ${ }^{2-4}$

La etiología de esta maloclusión está en una base maxilar superior ancha en relación a la base mandibular o por inclinaciones dentales posteriores hacia lingual y asimetrías mandibulares. En este tipo de maloclusión se observan las cúspides palatinas contactarán con las caras vestibulares inferiores. ${ }^{7-10}$

La prevalencia de mordidas cruzadas posteriores vestibulares es baja, siendo de 1 a 1,5\% ${ }^{9,12}$

El objetivo del tratamiento en dentición primaria y mixta temprana es equilibrar la oclusión, favoreciendo un desarrollo armónico y reducir la complejidad del tratamiento en dentición permanente, el cual puede llegar a ser muy invasivo involucrando ortodoncia y técnicas quirúrgicas. ${ }^{710,14,15}$

En general existen dos tipos de tratamiento para este tipo de maloclusión, dependiendo de su severidad y origen. Para casos poco severos puede realizarse la eliminación de interferencias oclusales mediante ajustes oclusales, desgastes selectivos y/o pistas directas de resina. Cuando la mandíbula tiene deficiencia transversal, particularmente si los molares mandibulares se encuentran con inclinación lingual, el abordaje es la expansión mandibular mediante a aparatos ortodóncicos, dependiendo de las exigencias de cada casa puede ser fija, como el Bi-Helix o removibles con tornillos de expansión. 3, 5, 7, 8, 10,15-19

Se presentan dos casos que corresponden a pacientes pediátricos con mordida cruzada posterior unilateral en dentición primaria o mixta temprana, quienes recibieron tratamiento de ortodoncia interceptiva en el postgrado de Odontopediatría de la UCV, logrando corrección exitosa de la maloclusión.

\section{Reporte de casos}

Se presentan a continuación dos casos de pacientes que acudieron con sus representantes, al post grado de Odontología Infantil, para ser rehabilitados bucalmente. Los representantes fueron informados del diagnóstico, la naturaleza de la enfermedad que padecían sus representados y su evolución natural, objetivos del tratamiento propuesto, y las alternativas de tratamiento. Aceptando la realización de las radiografías, fotografías y otros medios diagnósticos necesarios y la utilización de dichos record para la publicación del caso con fines académicos. Todo lo anteriormente expuesto mediante el consentimiento informado para atención Odontológica del paciente en el Postgrado de odontología infantil de la Facultad de Odontología de la UCV, aprobado por el Comité de Bioética de esta institución.

\section{Paciente $\mathbf{n}^{\circ} \mathbf{1}$}

Paciente masculino de 5 años de edad, aparentemente sano quien recibió alimentación materna durante un año, siendo exclusiva durante 6 meses. Al examen clínico extraoral se observa, que la forma de la cara es mesofacial y simétrica.

En el examen clínico intraoral se observan los tejidos blandos sin alteración, múltiples lesiones de caries activas cavitadas línea media dental superior desviada $3 \mathrm{~mm}$ hacia la derecha, normoclusión canina, escalón mesial suave molar y mordida cruzada posterior vestibular derecha. Los segundos molares inferiores presentaban inclinación lingual (Fig. 1)

Se realizó tratamiento integral rehabilitador e intervención ortodóncica mediante aparato fijo Bi Helix con bandas cementadas a segundos molares primarios. (Fig. 2)

La activación del Bi-Helix se realizó fuera de boca, considerando el ancho transversal molar fi- 


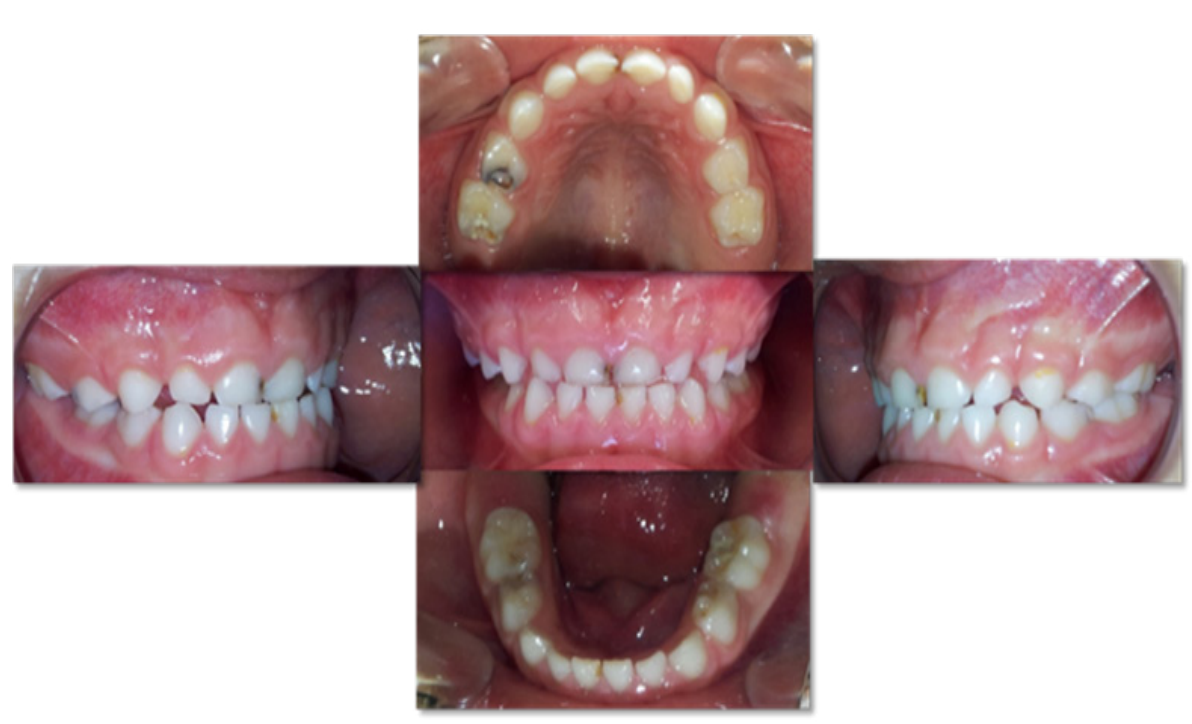

Fig. 1. Fotografías intraorales iniciales paciente $n^{-} 1$.

nal que se deseaba lograr incluyendo inclinación vestibular de los molares en los que se cementarían las bandas.

El control al mes de instalación demostró corrección de la mordida cruzada vestibular con apropiados de las cúspides palatinas de los molares superiores y los molares inferiores. Se mantuvo el aparato cuatro meses posteriormente como retención para el movimiento logrado.

En este caso se observó enderezamiento de los molares inferiores como el principal factor que permitió la corrección.

Lamentablemente no puede determinarse estos aspectos por falta de regitros clínicos finales.

\section{Paciente $\mathbf{n}^{\mathbf{0}} \mathbf{2}$}

\section{Datos del paciente}

Paciente masculino de 4 años de edad, acude a conwsulta acompañado de su representante, quien refiere traerlo a consulta por tener múltiples lesiones de caries. Como deato de interés, el paciente recibió alimentación materna du- rante 2 años, siendo exclusiva hasta los 4 meses.

$\mathrm{Al}$ examen extra bucal se observa que la forma de la cara es mesofacial, preservando la simetría.

$\mathrm{Al}$ examen intrabucal se observan múltiples lesiones da caries cavitadas con compromiso pulpar, absceso dentoalveolar crónico fistulizado en incisivos primarios superiores, relación canina primaria normoclusión, relación molar derecha escalón mesial y mordida cruzada posterior vestibular izquierda con perdida de la dimensión vertical por caries en los molares. (Fig. 4)

El tratamiento realizado fue rehabilitación bucal integral incluyendo de corona de acero inoxidable en: $74,75,85$, exodoncias de 52, 52, 61,62, confección de mantenedor de espacio superior removible con plano de levantamiento de mordida anterior.

Se realizó la restauración de la dimensión vertical y el equilibrio de oclusión a expensas de la colocación de coronas de acero inoxidable en los molares inferiores severamente afectados por caries y de pistas directas de resina en los molares primarios superiores. (Fig 5 y 6 )

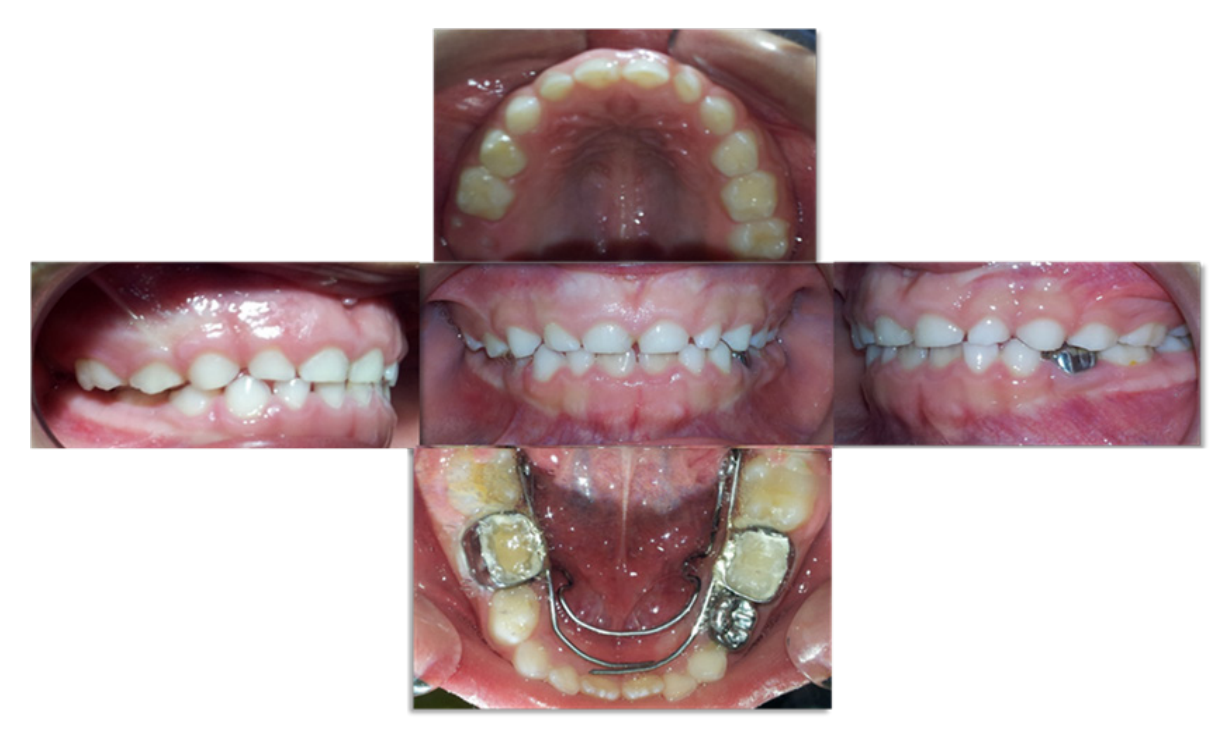

Fig. 2. Fotografías clínicas intraorales Paciente \#1 al instalar el Bi-Helix. 


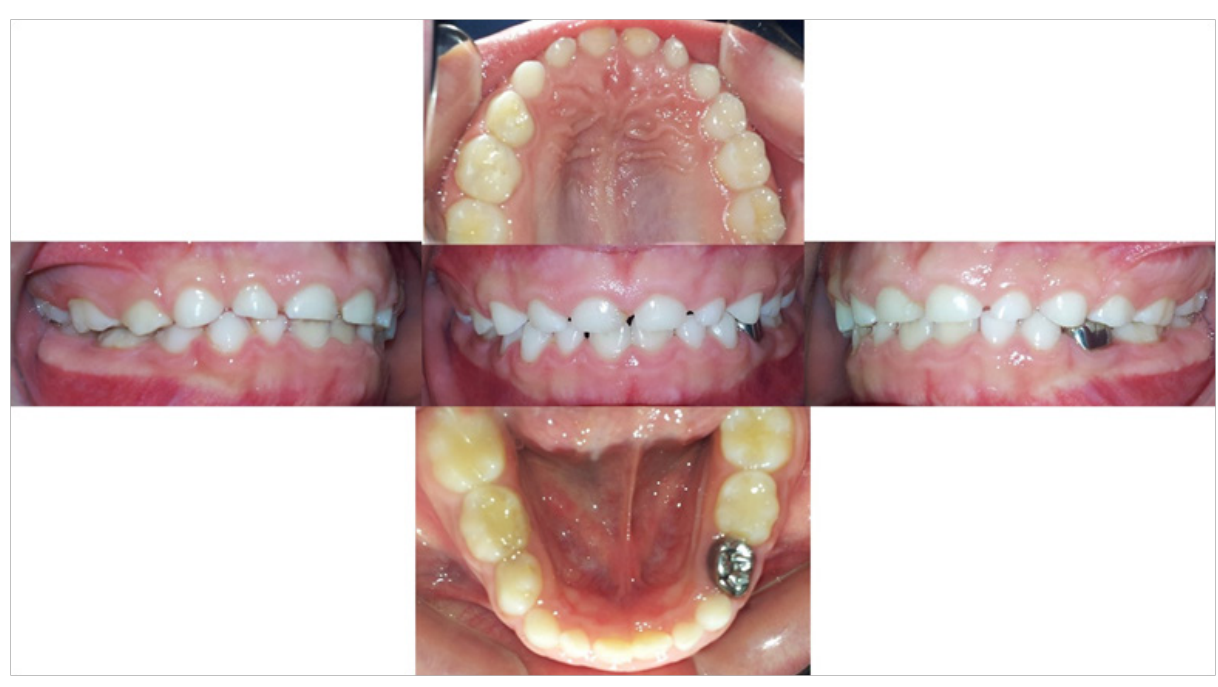

Fig. 3. Control a los 3 meses posterior a la instalación del Bi-Helix paciente $n^{-} 1$.

En el Caso 2, no hubo modificación en el ancho intermolar e intercanino inferior, siendo que la corrección de la mordida cruzada vestibular se realizó a expensas de modificaciones anatómicas (pistas) en los molares superiores y a la restauración anatómica (coronas de acero inoxidable) de los molares inferiores.

\section{Discusión}

Las mordidas cruzadas posteriores vestibulares son una maloclusión poco frecuente en dentición primaria y mixta temprana. Sin embargo, se han descrito los beneficios de su tratamiento temprano. 3-12,17-21 Hellman en 1921 afirmó “tan importante es que la cúspide mesiovestibular se encuentre en clase I de Angle como que la cúspide mesiopalatina se encuentre en la fosa central del molar inferior. ${ }^{4,5}$

Diferentes estudios han descrito que la etiología de la mordida cruzada vestibular unilateral posterior incluye un componente funcional de base, pudiendo ser producto de interferencias oclusales y/o volcamientos de los molares inferiores hacia lingual. ${ }^{3,10}$

En el presente trabajo se reportan dos casos de mordida cruzada vestibular posterior. En el caso del primer paciente la mordida cruzada vestibular posterior se debía a un volcamiento hacia lingual de los molares primarios inferiores del lado derecho. $Y$ en el caso del segundo paciente esta maloclusión se debía a interferencias oclusales debido a la perdida de dimensión vertical producida por las múltiples lesiones de caries. En ambos caso se observó una desviación de la línea media en el sentido contrario de hacia donde se cruzaba la mordida. En consonancia con lo reportado en la literatura. ${ }^{3,7}$

Existen varias modalidades de tratamiento para la intercepción de la mordida cruzada posterior vestibular en dentición primaria, eliminando las interferencias oclusales, bien sea con desgastes selectivo, con pistas de resina directa o mediante

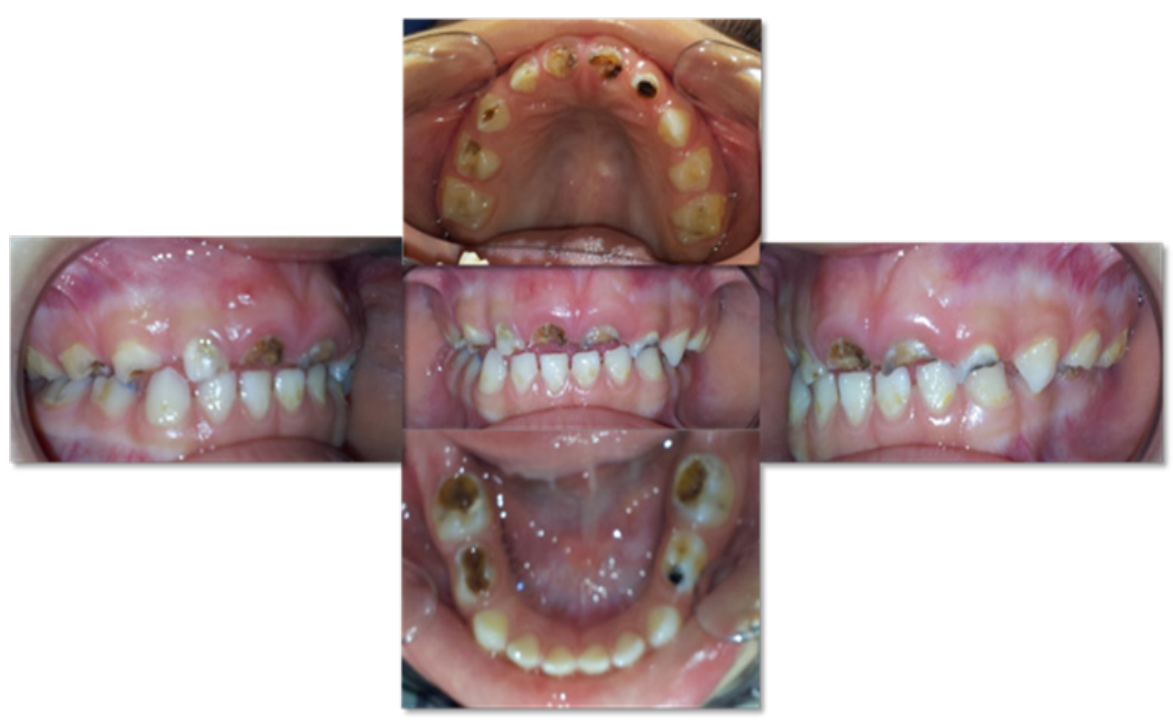

Fig. 4. Fotografías iniciales paciente $n^{\underline{0}} 2$. 


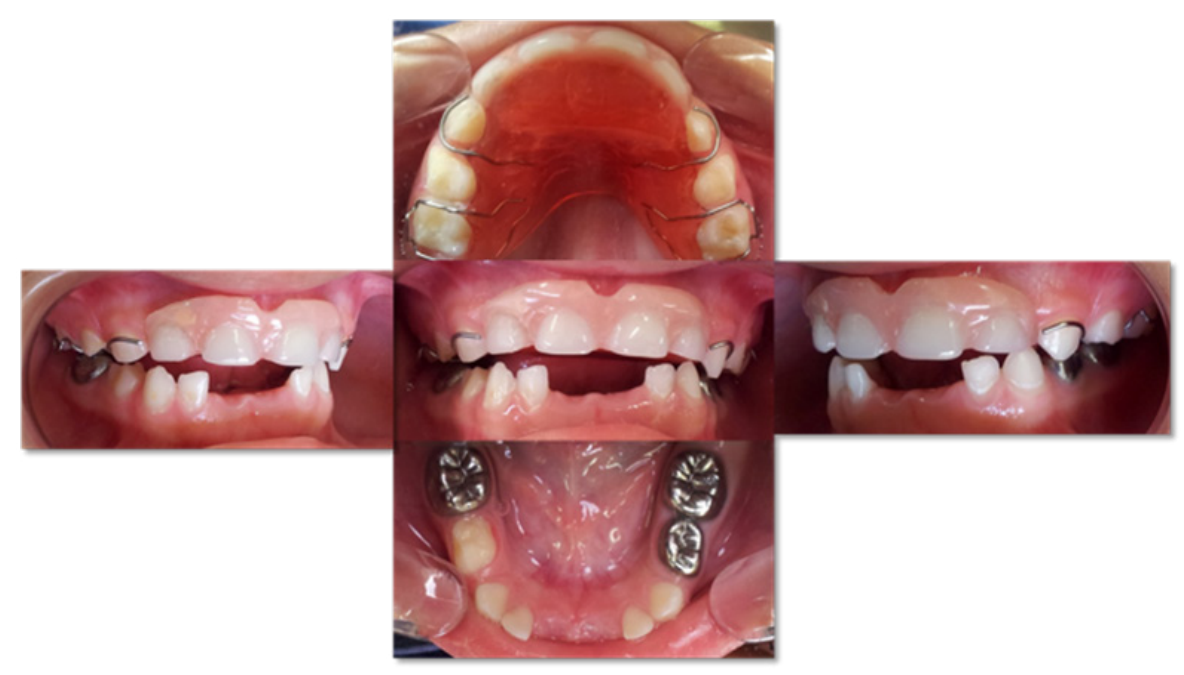

Fig. 5. Fotografías clínicas intraorales finales paciente $n^{-} 2$. Mantenedor de espacio y pistas directas de resina.

un volcamiento de los molares mandibulares hacia vestibular con aparatos removibles o fijos. ${ }^{3,5,7-}$ $10,15,18,19$

Los desgastes selectivos y las pistas de resina directa, representan una excelente opción para logran corregir las mordidas cruzadas del tipo funcional, para así lograr redirigir la mandíbula a su posición correcta en relación y oclusión céntrica. Siendo un tratamiento con resultados casi inmediatos. Esta alternativa de terapéutica fue utilizada exitosamente en el caso $\mathrm{n}^{\mathrm{o}} 2$ incluyendo rehabilitación de caries, reconstrucción del plano de oclusión e instalación de mantenedor de espacio. ${ }^{7,5,10}$
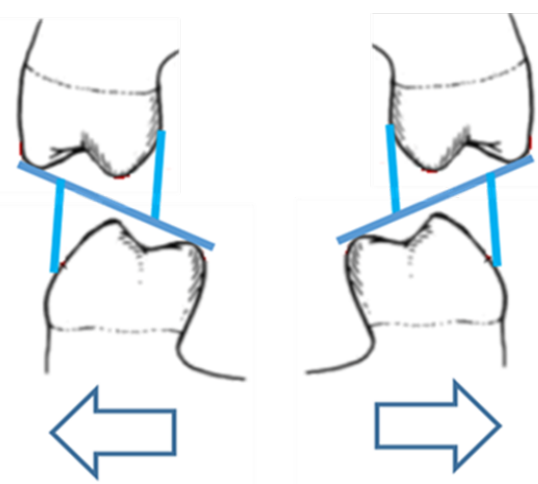

Fig. 6. Dibujo esquemático representativo de las inclinaciones de las pistas directas de resina para tratar mordidas cruzadas vestibulares.
El Bi-helix es un aparato diseñado especifica mente para logra un volcamiento de los molares inferiores y así estimular un remodelado óseo de la mandíbula permitiendo así lograr una adecuada relación y engranaje molar. $8,9,17,19$

El caso $\mathrm{n}^{\mathrm{o}} 1$ fue tratado de forma exitosa utilizando $\mathrm{Bi}$ hélix, incluyendo rehabilitación previa de caries.

En la literatura se encuentra que existe un porcentaje de pacientes con mordidas cruzadas posteriores funcionales que se autocorrigen durante el recambio dentario, sin embargo no se puede garantizar una auto corrección en todos los casos, y esto trae como consecuencia una mordida cruzada posterior en dentición permanente que sí puede conllevar a tratamientos más complejos, y con mayor riesgo a recidiva. ${ }^{7,8,10}$

Es importante el abordaje de estas maloclusiones en dentición primaria y mixta temprana, ya que el tratamiento en estas primeras etapas constituye sea sencillo y muy bien tolerado por el paciente. Esto evitara futuras deformidades faciales, proporcionando una adecuada posición de los cóndilos y equilibrio neuromuscular. ${ }^{7,10}$

\section{Conclusión}

Las mordidas cruzadas vestibulares posteriores en dentición primaria y mixta temprana pueden deberse a interferencias oclusales, desviaciones mandibulares e inclinaciones dentales inapropiadas, como en los casos presentados.

La corrección de la mordida cruzada posterior unilateral vestibular en dentición primaria y mixta temprana, puede lograrse de forma eficiente por medio de la eliminación de interferencias oclusales, utilización de pistas directas de resina o expansión inferior con aparatos de tipo Bi-helix. 


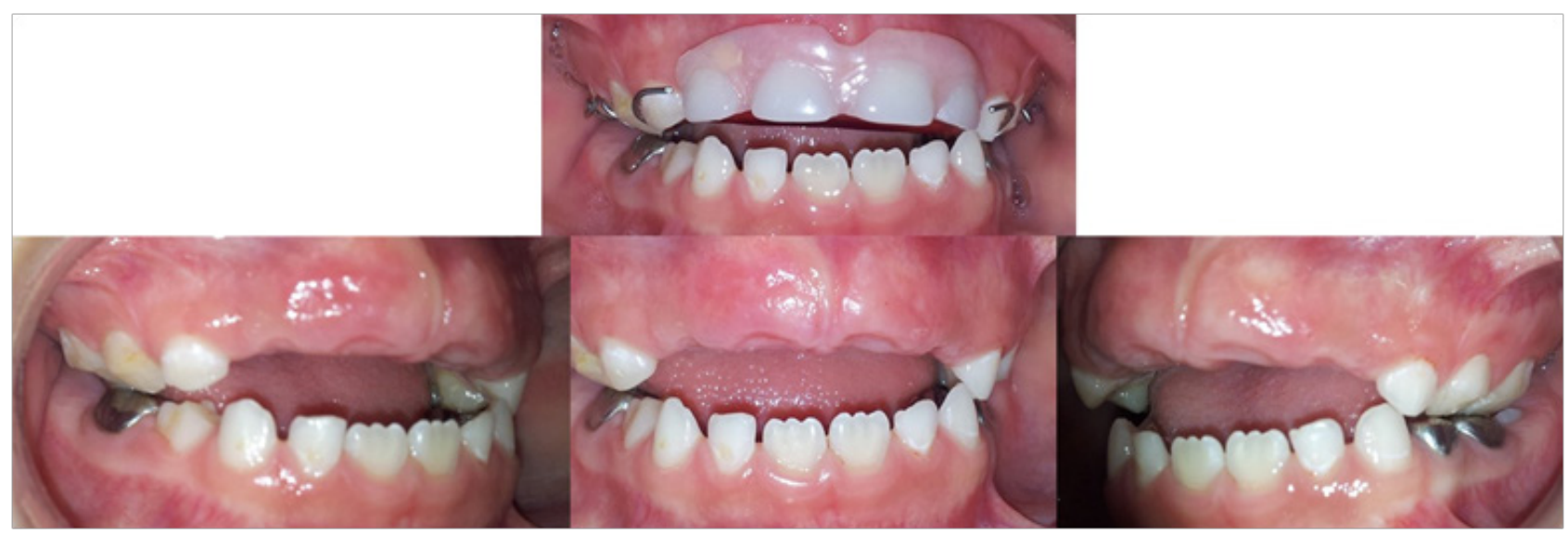

Fig. 7. Control a los 3 meses de rehabilitación paciente $n^{\underline{0}} 2$.

\section{Referencias bibliográficas.}

1. Saturno D' Escrivan L, Torres MC. Ortodoncia en denticion Mixta Caracas: Amolca; 2007.

2. Proffit WR, Ackerman J. The characteristics of maloclusion: A modern approach to classification and diagnosis. Am J Orthod. 1969; 55: 443-454.

3. Malandris M, Mahoney Ek. Aettiology, diagnosis and treatment of posterior cross-bites in the primary dentition. International J Ped Dent. 2004; 14: 155-66.

4. Castañer A. Med. Oral patol. oral cir.bucal. [Online].; 2006. Disponible en: http://scielo.isciii.es/scielo.php?script=sci_arttext\&pid=S1698-69462006000200022\&lng=es.

5. Padilla M, Tello L, Hernández J. Enfoque temprano de las maloclusiones transversales, diagnóstico y tratamiento. Revisión de la literatura. Rev Estomat. 2009; 17: 30-7.

6. Dimberg L, Lennarstsson B, Söderfeldt B, Bondermark L. Malocclusions in children at 3 and 7 years of age: a longitudinal. Eu J Orthod. 2013; 35: 131-7.

7. Kisling E. Occlusal interferences in the primary dentittion. ASDC J Dent Child. 1981; 43: 151-91.

8. Valencia R. Treatment of unilateral buccal crossbites in the primary, early mixed and permanet dentitions: Case reports. J Clin Ped Dent. 2007; 31: 214-8.

9. Nojima K, Takaku S, Murase C, Nishii Y, Sueshi K. A Case Report of Bilateral Brodie Bite in Early Dentition Using Bonded Constriction Quad Helix Appliance. Case report. Bull Tokyo Dent Coll. 2011; 52: 39-46.

10. Dos Santos R, Isper Garbin A, Saliba Garbin A. Early Correction of Malocclusion Using Planas Direct Tracks.Case Report. Case Rep Dent. 2013; 2013: 395784. Published online 2013 Sep 3. Consultado 03 de mayo 2015. http://www.ncbi. nlm.nih.gov/pmc/articles/PMC3776552/

11. Thilander B, Pena L, Infante C, Parada SE, de Mayorga C. Prevalence of malocclusion and orthodontic treatment need in children and adolescentes in Bogota, Colombia. An epiddemiological study to different stages. Eu J Orthod. $2001 ; 23$ : 153-62.

12. Galarza L, Torres V. Síndrome de Brodie en denticion mixta temprana. Tratado con aparatologia removible adhesiva. Resporte de un caso. Odontol. Sanmarquina. 2008; 11: 88-91.

13. FUNDACREDESA. Estudio sobre la oclusion dental Indice IPTO. En Estudio Nacional de crecimiento y desarrollo Humano de la Republica de Venezuela. Caracas; 1996.

14. Leighton B. Early recognition of normal occlusion. In the biology of occlusal development University of Michigan: Ed. Macnamara JA Jr Craniofacial series. Center for human growth and development; 1997.

15. Planas P. Rehabilitación Neuro-Oclusal (RNO) Barcelona: Actualidades Médico Odontológicas Latinoamérica, C.A; 2000. 16. Pignataro Neto G, Puppin-Rontani RM, Rodriguez Garcia RCM. Changes in the masticatory cycle after treatment of posterior crossbites in children aged 4 to 5 years. AJO. 2007 Abril; 131: 464-72.

17. Langbert B, Kazuhito A, Miner M. Asimetrias transversales esqueletales y dentarias en pacientes con mordida cruzada posterior unilateral. AJO. 127. 2005; 127: 6-15.

18. Mata J, Zambrano F, Quirós O, Farias M, Rondón S, Lerner H. Expansión rápida de maxilar en Maloclusiones transversales: Revisión Bibliográfica. Revista Latinoamericana de Ortodoncia y Odontopediatria. [Online].; 2007. Disponible 
en: https://www.ortodoncia.ws/publicaciones/2007/art11.asp. Consultada 12 de julio 2015.

19. Lippold C, Stamm T, Meyer U, Végh A, Moiseenko T, Danesh G. Early treatment of posterior crossbite- a randomised clinical trial. Disponible en http://www.trialsjournal.com/content/14/1/20. 2013;: 14:20. Consultada 12 de julio 2015.

20. Ugalde F. Clasificación de la maloclusión en los planos anteroposterior, vertical y transversal. ADM. 2007; 97-109.

21. Abraham JE, Alexander SA. Overview of anterior and posterior crossbites in children. NYSDJ. Ped Dent. 1997; 3: 36-38.

Recibido: 08-01-16

Aceptado: 09-05-16

Correspondencia: Jerusalén E. Mata T.

jerusalenmata@gmail.com. 